\title{
Hydrogen Bond Cooperativity and the Three-Dimensional Structures of Water Nonamers and Decamers**
}

\author{
Cristóbal Pérez, Daniel P. Zaleski, Nathan A. Seifert, Berhane Temelso, George C. Shields,* \\ Zbigniew Kisiel,* and Brooks H. Pate*
}

\begin{abstract}
Broadband rotational spectroscopy of water clusters produced in a pulsed molecular jet expansion has been used to determine the oxygen atom geometry in three isomers of the nonamer and two isomers of the decamer. The isomers for each cluster size have the same nominal geometry but differ in the arrangement of their hydrogen bond networks. The nearest neighbor $\mathrm{O}^{-} \mathrm{O}$ distances show a characteristic pattern for each hydrogen bond network isomer that is caused by three-body effects that produce cooperative hydrogen bonding. The observed structures are the lowest energy cluster geometries identified by quantum chemistry and the experimental and theoretical $\mathrm{O}-\mathrm{O}$ distances are in good agreement. The cooperativity effects revealed by the hydrogen bond $\mathrm{O}-\mathrm{O}$ distance variations are shown to be consistent with a simple model for hydrogen bonding in water that takes into account the cooperative and anticooperative bonding effects of nearby water molecules.
\end{abstract}

W ater is the most important solvent in chemistry and biology and displays unique physical properties related to its ability to form hydrogen bonds. ${ }^{[1,2]}$ A special feature of the hydrogen bond is its cooperativity, i.e., the fact that the local hydrogen bond strength is influenced by the neighboring water molecules. Hydrogen bond cooperativity affects the vibrational spectrum of the $\mathrm{O}-\mathrm{H}$ stretches, ${ }^{[3]}$ properties of low-temperature liquid phases ${ }^{[4]}$ and site-specific reaction rates for chemical processes like acid dissociation in water

[*] Dr. C. Pérez, Dr. D. P. Zaleski, N. A. Seifert, Prof. B. H. Pate Department of Chemistry, University of Virginia McCormick Rd., Charlottesville, VA 22904-4319 (USA)

Dr. B. Temelso, Prof. G. C. Shields

Dean's Office, College of Arts and Sciences, and

Department of Chemistry, Bucknell University

Lewisburg, Pennsylvania 17837 (USA)

Prof. Z. Kisiel

Institute of Physics, Polish Academy of Sciences

Al. Lotników 32/46, 02-668 Warszawa (Poland)

[**] This work was supported by U.S. National Science Foundation (NSF) grants CHE-0960074 and CHE-0848827; the Institute of Physics, Polish Academy of Sciences; and NSF grants CHE$0116435, \mathrm{CHE}-0521063$, and CHE-0849677 as part of the MERCURY high-performance computer consortium (www.mercuryconsortium.org). This research used the NSF TeraGrid resources provided by the Texas Advanced Computing Center (TACC) under grant TGCHE090095 and resources of the National Energy Research Scientific Computing Center, which is supported by the Office of Science of the U.S. Department of Energy under contract DE-AC02$05 \mathrm{CH} 11231$.

Supporting information for this article is available on the WWW under http://dx.doi.org/10.1002/anie.201407447. clusters. ${ }^{[5]}$ Quantum chemistry studies of small water clusters play a key role in developing model potentials that include the three-body effects that produce cooperative hydrogen bonding. ${ }^{[6-10]}$ The three-body effects can make sizable contributions to the total binding energy of small clusters and have been shown to account for about $20 \%$ of the total binding energy for several cluster geometries. ${ }^{[6]}$ Cooperative hydrogen bonding affects several physical and geometrical parameters including the local hydrogen bond strength, the $\mathrm{O}-\mathrm{H}$ stretch vibrational frequency, the $\mathrm{O}-\mathrm{O}$ nearest neighbor distances, and the donor $\mathrm{O}-\mathrm{H}$ bond length deformation. ${ }^{[6-13]}$

Previous experimental studies of the water octamer, nonamer, and decamer have used vibrational spectroscopy to characterize the cluster structure. One experimental approach uses benzene to "tag" the neutral water cluster so that powerful ultraviolet (UV) and UV-infrared doubleresonance techniques can be used to measure the vibrational spectrum of size-specific water clusters. These experiments demonstrated the presence of the $\mathrm{S}_{4}$ and $\mathrm{D}_{2 \mathrm{~d}}$ water octamers $^{[14]}$ and identified three isomers of the water nonamer. ${ }^{[15]}$ However, it was unclear if these isomers resulted from changes in the nonamer geometry or the attachment point of the benzene ring. Vibrational spectra of water clusters have also been obtained using size-selected crossed molecular beams. ${ }^{[16]}$ The $\mathrm{S}_{4}$ and $\mathrm{D}_{2 \mathrm{~d}}$ octamer isomers were observed and there was evidence for more than one isomer of the water nonamer. The interpretation of the $\mathrm{O}-\mathrm{H}$ stretch fundamental spectrum of the water decamer indicated a "butterfly" structure instead of the pentagonal prism which has been shown in several theoretical studies ${ }^{[17-19]}$ to be the lowest energy structure.

Here we use broadband molecular rotational spectroscopy to obtain accurate structures of the oxygen framework geometry for isomers of the water nonamer and decamer. ${ }^{[20]}$ The nonamer and decamer structures observed in the pulsed jet expansion are related to the $S_{4}$ and $D_{2 d}$ water octamer structures and the theoretical structures for each cluster size are shown in Figure 1. The experimental challenge is to distinguish isomers that have essentially the same heavy atom structure and the same number of hydrogen bonds, but with different hydrogen bond donor-acceptor patterns. The key to the experimental identification of isomers is the distortion of the oxygen atom framework that results from hydrogen bond cooperativity in the three-dimensional structures.

Rotational spectra of two different water samples were measured in the $2-8 \mathrm{GHz}$ frequency range using a chirpedpulse Fourier transform microwave spectrometer. ${ }^{[21]}$ One sample was pure water and this spectrum was used to identify rotational spectra of clusters composed entirely from the 


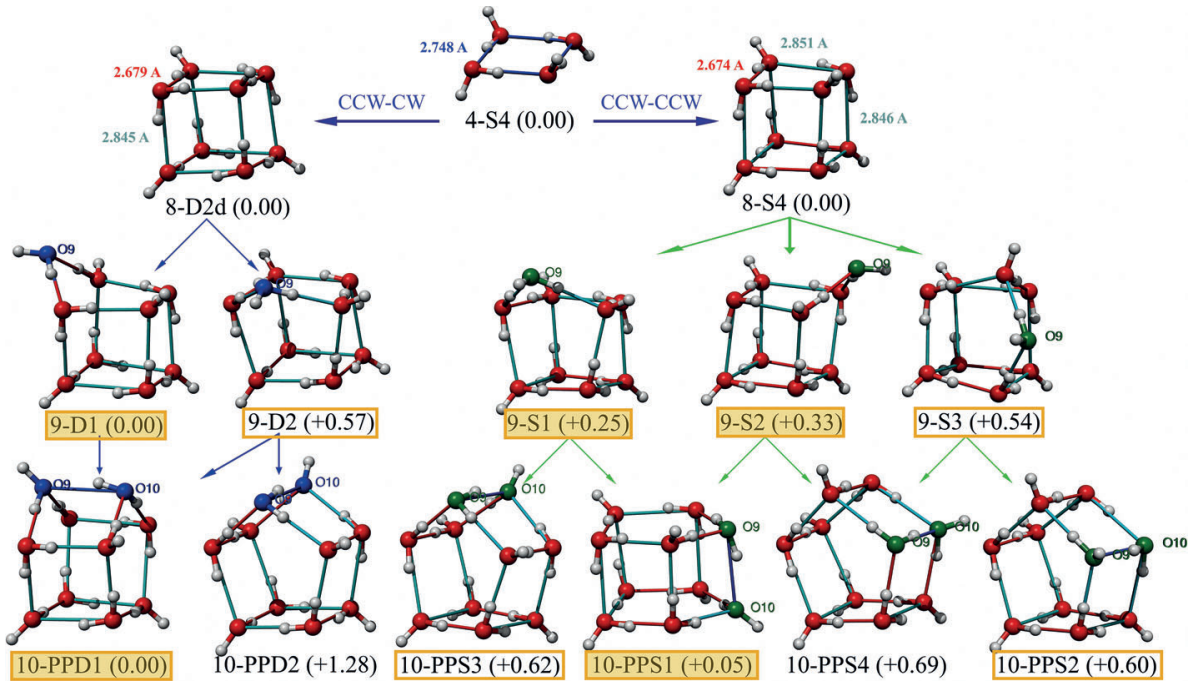

Figure 1. The top row shows the two water octamers with stacked tetramer units that differ in the direction of the hydrogen bond orientation (counterclockwise-clockwise and clockwise-clockwise). Five distinct nonamers are generated by inserting the ninth water (O9) as single-donor singleacceptor to the unique edges of the water octamer, two for $\mathrm{D}_{2 \mathrm{~d}}$ and three for $\mathrm{S}_{4}$. The bottom row shows the insertion of the tenth water molecule $(\mathrm{O} 10)$ into the nonamers to generate six water decamers. Short and long distances are shown as red and cyan bonds. In parentheses, the relative energies are given in units of $\mathrm{kcal} \mathrm{mol}^{-1}$. All calculations are performed at the RI-MP2/CBS level of theory. The encircled (orange) labels indicate that the cluster was observed experimentally; the shaded labels indicate that a full assignment of their singly ${ }^{18} \mathrm{O}$-substituted isotopologues was performed.

$\mathrm{H}_{2}{ }^{16} \mathrm{O}$ water isotope. A total of five water nonamer clusters and four water decamer clusters were detected. Of the four water decamers identified in the pure water spectrum, three show the effects of tunneling with patterns similar to the water hexamer prism. ${ }^{[20]}$ The second sample was spiked with $12.5 \%$ of $\mathrm{H}_{2}{ }^{18} \mathrm{O}$. The molecular rotational spectra of water clusters with a single $\mathrm{H}_{2}{ }^{18} \mathrm{O}$ positional substitution were identified for three of the water nonamer clusters and two of the water decamer clusters. The tunneling splitting for the water decamers is quenched in the substituted $\left(\mathrm{H}_{2}{ }^{16} \mathrm{O}\right)_{9}$ $\left(\mathrm{H}_{2}{ }^{18} \mathrm{O}\right)$ spectra. Small frequency regions of the broadband rotational spectrum are presented in Figure 2 to show the isomer identification and the effect of isotopic substitution.

The experimental oxygen atom framework geometry is obtained from the analysis of the rotational constants of the $\left(\mathrm{H}_{2}{ }^{18} \mathrm{O}\right)\left(\mathrm{H}_{2}{ }^{16} \mathrm{O}\right)_{N-1}$ isotopologues for each cluster and the results are shown in Figure 3. The initial analysis uses Kraitchman's equations ${ }^{[22]}$ to determine the position of each oxygen atom in the rotational principal axis system individually. This method can only determine the magnitude of the atom positions and the signs of the coordinates are selected by comparison to the theoretical structures. The oxygen atom positions obtained from the Kraitchman analysis are shown by the solid spheres in Figure 3. Once the basic oxygen atom framework geometry was established, further structural refinement was performed using the $r_{m}{ }^{(1)}$ model. ${ }^{[23,24]}$ This analysis accounts for zero-point vibrational averaging effects and produces a structure that is closer to the equilibrium geometry than a Kraitchman substitution structure. Therefore, the $r_{m}{ }^{(1)}$ geometry can be more directly compared with ab initio equilibrium geometries. The $r_{m}{ }^{(1)}$ analysis of the oxygen atom framework geometry requires constraints on the other structure parameters, the water monomer geometries and their relative orientations, and these are fixed to the values from the theoretical structures. The $\mathrm{r}_{\mathrm{m}}{ }^{(1)}$ cluster structures are shown in Figure 3. It is seen that the oxygen atom positions are nearly the same from the two analysis methods. Previous studies of the water hexamer and heptamer that included vibrational averaging in the theoretical structures have shown that the oxygen atom framework geometry does not show large changes from vibrational averaging and that rotational spectroscopy isotopic substitution studies are in quantitative agreement with the theoretical results.

The lowest energy isomers for the water nonamer shown in Figure 1 are formed by adding a water molecule to a distinct edge of the octamer as discussed previously. ${ }^{[15]}$ The $\mathrm{D}_{2 \mathrm{~d}}$ octamer has only two distinct edges whereas the $\mathrm{S}_{4}$ octamer has three. A more complete description of these isomers is presented in the Supporting Information (SI) and Figures S14-S16. The resulting isomers have the same nominal oxygen atom framework, a ring pentamer stacked on a ring tetramer, but with different hydrogen bond networks that give characteristic distortions of the nearest-neighbor $\mathrm{O}_{-}^{-}$ $\mathrm{O}$ distances.

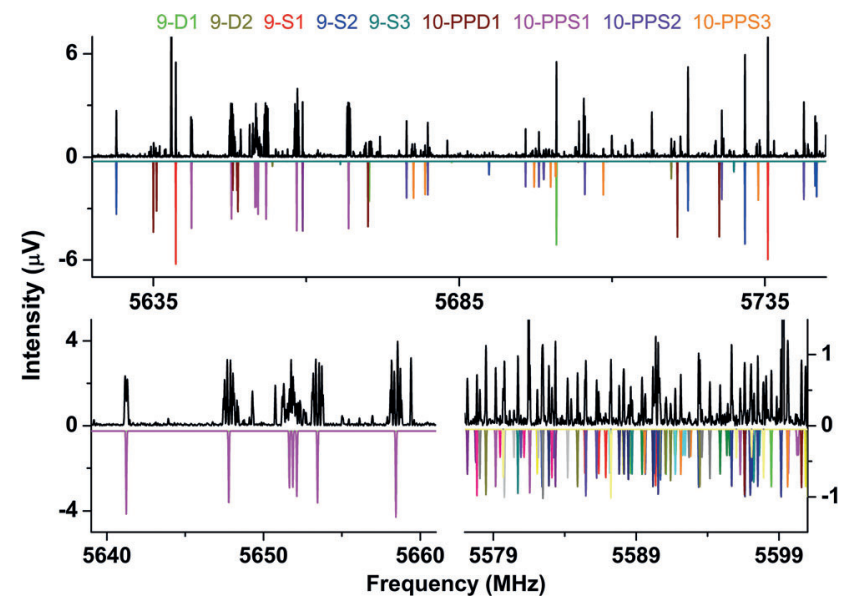

Figure 2. The top panel shows a region of the experimental CP-FTMW spectrum of water in a neon backing gas. The black trace is the experimental spectrum, and the colored traces are spectrum simulations of the observed water clusters $\left(T_{\text {rot }}=1.5 \mathrm{~K}\right)$. The bottom panel (left) shows tunneling splitting observed in decamer 10-PPS1. The spectrum has been fit to the line centers (magenta). The bottom panel (right) is a $400 x$ magnification demonstrating that all ${ }^{18} \mathrm{O}$ decamer species are fully resolved. 

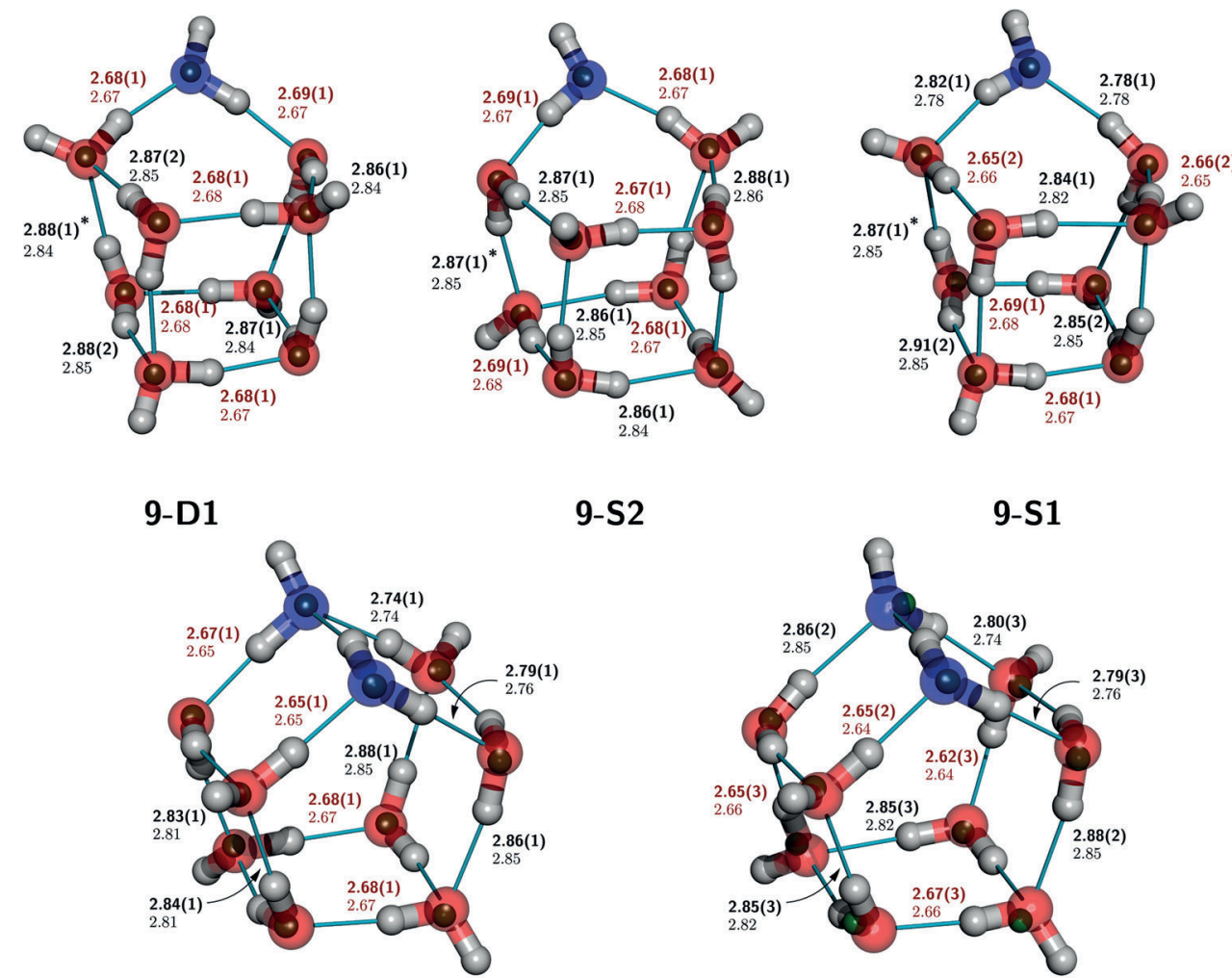

9-S2
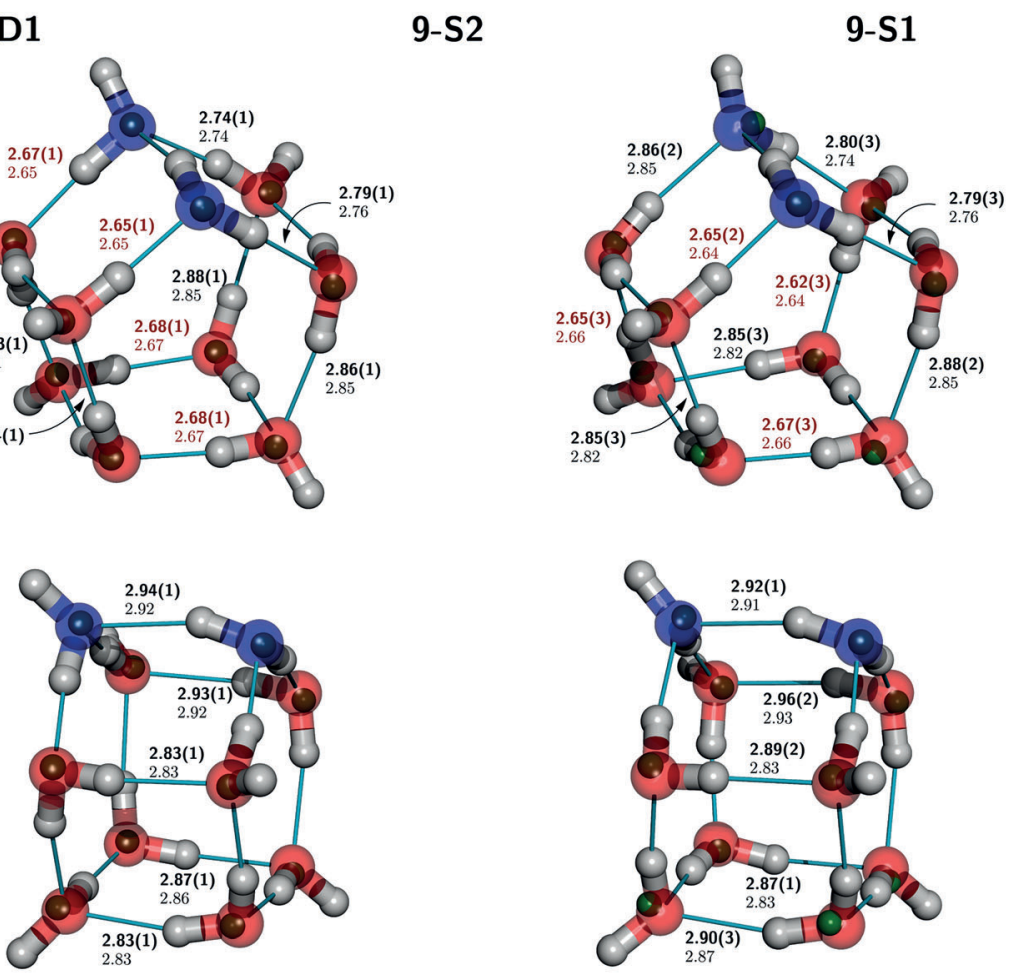

10 - PPD1

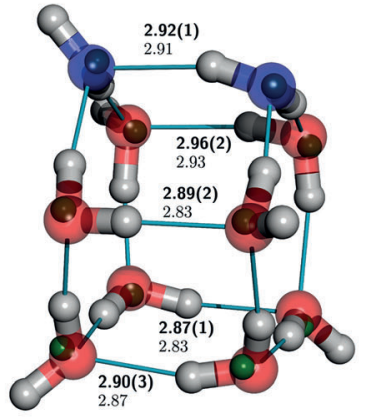

10 - PPS1

Figure 3. Comparison of the two experimental structures determined by ${ }^{18} \mathrm{O}$ substitution: $r_{m}{ }^{(1)}$ (complete water molecules) and $r_{s}$ (green spheres). In the marked distances $(\AA)$ the top value is from the leastsquares $r_{m}{ }^{(1)}$ geometry, whereas the bottom is the calculated RI-MP2/aug-cc-pVDZ equilibrium value. The color coding distinguishes short (red) and long (black) distances. In the nonamers the calculated pillar distances between the top pentagon and the base tetragon were set equal in the fits (asterisked). The blue water units correlate with the $\mathrm{O} 9$ and $\mathrm{O} 10$ units marked in Figure 1. The foreground pentamer rings in the two decamers are identical (middle row), whereas the background pentamer rings have opposite (10-PPD1) and equal (10-PPS1) directionality of the $\mathrm{H}$-bonding networks. The middle and bottom rows show the $\mathrm{O}-\mathrm{O}$ distances in the pentamer rings and the interring distances, respectively.

The experimental oxygen atom framework structures are in excellent agreement with the three lowest energy theoretical structures as shown in Figure 3, in which the experimental and theoretical nearest-neighbor $\mathrm{O}-\mathrm{O}$ distances are given. The experimental rotational constants of the two less abundant isotopically pure water nonamers, for which the signal-to-noise ratio is insufficient to determine the oxygen atom framework structures from the isotopic measurement, are consistent with the theoretical values for the higher energy 9-D2 and 9-S3 nonamer isomers (see Table S1). The relative abundances of the five water nonamers in the molecular beam have been estimated from the transition intensities using the theoretical values of the dipole moments and are approximately 1 (9-D1): 1 (9S1):0.66 (9-S2):0.25 (9S3):0.1 (9-D2). The observed relative populations are in general agreement with the relative energies from quantum chemistry shown in Figure 1.

The low-energy decamer structures can be derived from the five nonamer structures by adding a second water molecule across a face of the parent octamer structure. The six structures in Figure 1 are the lowest energy isomers of the 102 possible topological isomers for this oxygen atom framework geometry. ${ }^{[17]}$ The structures 10-PPD1 and 10-PPS1 are the lowest energy isomers in each family and have been identified in several previous theoretical studies. ${ }^{[17-19]}$ They can also be viewed as the stacking of two ring structures, the lowest energy geometry for the water pentamer, ${ }^{[25]}$ in either clockwiseclockwise (10-PPS1) or clockwise-counterclockwise (10PPD1) orientations of the ring $\mathrm{H}$ bond network. Again, a distinctive pattern for the oxygen atom framework distortion is produced for each topological isomer.

As for the nonamers, there is excellent agreement in the characteristic nearestneighbor $\mathrm{O}-\mathrm{O}$ distance variations for the two decamers, for which quantitative oxygen atom framework geometries were obtained. Oxygen atom framework structures were obtained for the two highest population species in the pulsed supersonic expansion and, as shown in Figure 3, are the 10-PPD1 and 10-PPS1 isomers. The two lower population clusters are tentatively assigned to the 10-PPS2 and 10-PPS3 structure based on a comparison of experimental and theoretical rotational constants for the isotopically pure decamers. As for the nonamers, the relative populations of the decamer isomers [1 (10-PPD1):1 (10- 
PPS1): 0.2 (10-PPS2):0.2 (10-PPS3)] in the molecular beam are consistent with the relative energies from quantum chemistry (Figure 1).

The issue of hydrogen bond cooperativity in water clusters has received much theoretical attention since the experimental work on the structures of the water dimer and the "2D" ring isomers of the smaller water clusters $(n=3-5)$ showed a monotonic, and approximately exponential, decrease in the $\mathrm{O}-\mathrm{O}$ nearest-neighbor distance as the cluster size increases. ${ }^{[25]}$ For the three-dimensional structures reported in this work, in which all faces of the nonamers and decamers are either ring tetramers or pentamers, a different kind of hydrogen bond cooperativity is required to explain the characteristic and sizeable distortions to the $\mathrm{O}-\mathrm{O}$ distances.

As an example of the importance of cooperativity, we have calculated the increase in binding energy of the water octamer isomers associated with hydrogen bond cooperativity. The geometry distortion of the $\mathrm{S}_{4}$ tetramers ${ }^{[26]}$ from their square geometry produces significant stabilization of the $\mathrm{D}_{2 \mathrm{~d}}$ and $\mathrm{S}_{4}$ octamers ${ }^{[14]}$ formed by stacking of the tetramers. This stabilization energy has been estimated by calculating the energy of the cluster when the $\mathrm{S}_{4}$ tetramers are constrained to the square tetramer structure and the distance between the rings is optimized. The minimum energy structure occurs for a ring separation of $2.99 \AA$ and $3.01 \AA$ and stabilizes the complex by $12.4 \mathrm{kcal} \mathrm{mol}^{-1}$ and $12.0 \mathrm{kcal} \mathrm{mol}^{-1}$ for the $\mathrm{D}_{2 \mathrm{~d}}$ and $\mathrm{S}_{4}$ octamers, respectively, at MP2/aVDZ level of theory, relative to separated $S_{4}$ tetramers. When full geometry relaxation is allowed, leading to the distortions of the oxygen atom framework, the ring separation is reduced to $2.85 \AA$ and the stabilization energy increases to $19.9 \mathrm{kcal}$ $\mathrm{mol}^{-1}$ at the MP2/aVDZ level of theory as shown in Figure S12.

A simple model to characterize the cooperative hydrogen bond effects in three-dimensional water structures that is based on known relationships between $\mathrm{O}-\mathrm{H}$ stretch vibrational frequencies and the donor-acceptor properties of the water molecule ${ }^{[14-16,27]}$ has been presented by Ohno et al. ${ }^{[28]}$ The model assumes the usual picture that each water molecule can participate in up to four hydrogen bonds (two as donor and two as acceptor). The "net cooperativity" that affects a local hydrogen bond is defined as the sum of the number of occupied hydrogen bond positions in the donor and acceptor water that lead to cooperative strengthening of the bond less the number of occupied "anticooperative" sites that weaken the hydrogen bond. Recent theoretical analysis of this model by Tainter and Skinner has shown that the cooperativity effects arise from three body interactions. ${ }^{[1,12]}$ The experimental $\mathrm{O}-\mathrm{O}$ nearest-neighbor distances for the five clusters analyzed in this work are compared to the net cooperativity in Figure 4. As a point of reference, the experimental $\mathrm{O}-\mathrm{O}$ nearest-neighbor distances in the cyclic water tetramer and pentamer, in which a single distance characterizes the structure, are $2.79 \AA^{[26]}$ and $2.76 \AA_{\text {, }}^{\text {,29] }}$ respectively, and both structures have a net cooperativity of 2 for all hydrogen bonds.

Molecular rotational spectroscopy has observed hydrogen bond network isomers of three-dimensional water nonamer and decamer clusters. These isomers have the same basic

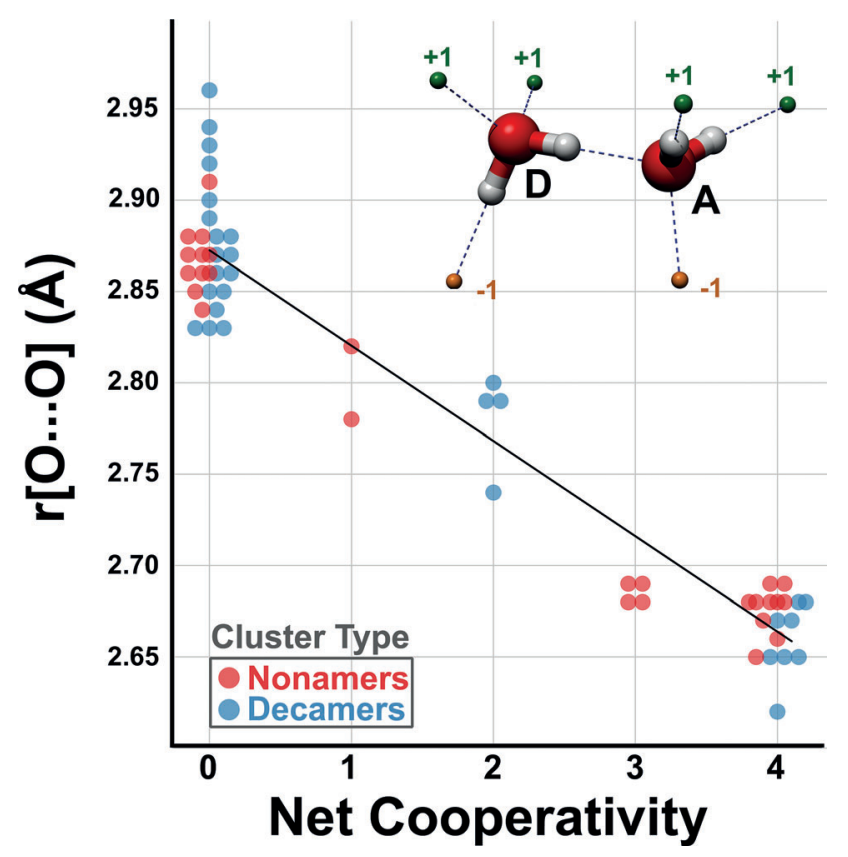

Figure 4. The correlation between experimental oxygen-oxygen distances and the net hydrogen bond cooperativity in the observed nonamers and decamers is shown. The net cooperativity of a donor-acceptor (DA) hydrogen bond is determined by calculating the sum of all cooperative neighboring hydrogen bonds $(+1)$ and anticooperative hydrogen bonds $(-1)$, as shown in the top right diagram. Cooperativity values are slightly offset from their integral values to show detail.

geometry, but differ in the details of the hydrogen bond arrangements. Cooperativity in hydrogen bonding produces distinctive distortions in the oxygen atom framework geometries that lead to $\mathrm{O}-\mathrm{O}$ nearest-neighbor distances varying from about 2.65-2.90 $\AA$ and the experimental and theoretical geometries are in excellent agreement. The pattern of the geometry distortions can be easily explained using a simple model for net hydrogen bond cooperativity. A recent theoretical study has shown that the local hydrogen bond strength is correlated with the $\mathrm{O}-\mathrm{O}$ distances and that cooperative hydrogen bonding leads to variations in the hydrogen bond strength by a factor of two or more within a three-dimensional water cluster environment. ${ }^{[30]}$ As a result, the hydrogen bond network geometry can influence reaction rates of molecules "solvated" in a cluster geometry. ${ }^{[5]}$ The flexibility of the three-dimensional water structure to adopt several nearly isoenergetic isomers with distinctive hydrogen bond networks with minimal motion in the oxygen atoms is a special feature of water that allows it to tune the local solvation site energetics. The results from the present study provide new benchmark measurements for the development of empirical intermolecular potentials and other parameterized methods for water that can be used in molecular modeling of a wide range of important chemical and biological systems.

Received: July 22, 2014

Revised: September 11, 2014

Published online: October 27, 2014 


\section{Angewandte}

Keywords: cooperativity · hydrogen bonding . microwave spectroscopy · vibrational spectroscopy . water clusters

[1] R. Bukowski, K. Szalewicz, G. C. Groenenboom, A. van der Avoird, Science 2007, 315, 1249-1252.

[2] N. S. Nagornova, T. R. Rizzo, O. V. Boyarkin, Science 2012, 336, $320-323$.

[3] B. Auer, R. Kumar, J. R. Schmidt, J. L. Skinner, Proc. Natl. Acad. Sci. USA 2007, 104, 14215-14220.

[4] K. Stokely, M. G. Mazza, H. E. Stanley, G. Franzese, Proc. Natl. Acad. Sci. USA 2010, 107, 1301-1306.

[5] J.-L. Kuo, M. L. Klein, J. Chem. Phys. 2004, 120, 4690-4695.

[6] M. V. Kirov, G. S. Fanourgakis, S. S. Xantheas, Chem. Phys. Lett. 2008, 461, 180-188.

[7] Y. Wang, X. Huang, B. C. Shepler, B. J. Braams, J. M. Bowman, J. Chem. Phys. 2011, 134, 094509.

[8] C. J. Tainter, P. A. Pieniazek, Y.-S. Lin, J. L. Skinner, J. Chem. Phys. 2011, 134, 184501.

[9] K. Szalewicz, C. Leforestier, A. van der Avoird, Chem. Phys. Lett. 2009, 482, 1-14.

[10] V. Babin, G. R. Medders, F. Paesani, J. Phys. Chem. Lett. 2012, 3, $3765-3769$.

[11] C. J. Tainter, J. L. Skinner, J. Chem. Phys. 2012, 137, 104304.

[12] C. J. Tainter, Y. Ni, L. Shi, J. L. Skinner, J. Phys. Chem. Lett. 2013, 4, $12-17$.

[13] E. Miliordos, E. Aprà, S. S. Xantheas, J. Chem. Phys. 2013, 139, 114302.

[14] C. J. Gruenloh, J. R. Carney, C. A. Arrington, T. S. Zwier, S. Y. Fredericks, K. D. Jordan, Science 1997, 276, 1678-1681.
[15] C. J. Gruenloh, J. R. Carney, F. C. Hagemeister, T. S. Zwier, J. T. W. Iii, K. D. Jordan, J. Chem. Phys. 2000, 113, 2290-2303.

[16] U. Buck, I. Ettischer, M. Melzer, V. Buch, J. Sadlej, Phys. Rev. Lett. 1998, 80, 2578-2581.

[17] A. Lenz, L. Ojamäe, Phys. Chem. Chem. Phys. 2005, 7, $1905-$ 1911.

[18] R. M. Shields, B. Temelso, K. A. Archer, T. E. Morrell, G. C. Shields, J. Phys. Chem. A 2010, 114, 11725-11737.

[19] H. Kabrede, R. Hentschke, J. Phys. Chem. B 2003, 107, $3914-$ 3920.

[20] C. Pérez, M. T. Muckle, D. P. Zaleski, N. A. Seifert, B. Temelso, G. C. Shields, Z. Kisiel, B. H. Pate, Science 2012, 336, 897-901.

[21] C. Pérez, S. Lobsiger, N. A. Seifert, D. P. Zaleski, B. Temelso, G. C. Shields, Z. Kisiel, B. H. Pate, Chem. Phys. Lett. 2013, 571, $1-15$.

[22] J. Kraitchman, Am. J. Phys. 1953, 21, 17-24.

[23] Z. Kisiel, J. Mol. Spectrosc. 2003, 218, 58-67.

[24] J. K. G. Watson, A. Roytburg, W. Ulrich, J. Mol. Spectrosc. 1999, 196, 102-119.

[25] K. Liu, M. G. Brown, J. D. Cruzan, R. J. Saykally, Science 1996, $271,62-64$.

[26] J. D. Cruzan, M. R. Viant, M. G. Brown, R. J. Saykally, J. Phys. Chem. A 1997, 101, 9022-9031.

[27] J. Sadlej, V. Buch, J. K. Kazimirski, U. Buck, J. Phys. Chem. A 1999, 103, 4933-4947.

[28] K. Ohno, M. Okimura, N. Akai, Y. Katsumoto, Phys. Chem. Chem. Phys. 2005, 7, 3005-3014.

[29] K. Liu, M. G. Brown, J. D. Cruzan, R. J. Saykally, J. Phys. Chem. A 1997, 101, 9011-9021.

[30] M. Huš, T. Urbic, J. Chem. Phys. 2012, 136, 144305. 